\title{
ESTUDO CINÉTICO DA ADSORÇÃO DO CORANTE TÊXTIL ALARANJADO DE METILA EM BENTONITA ATIVADA TERMICAMENTE
}

\author{
F. R. SANTOS $^{1}$, H. C. O. BRUNO ${ }^{1}$ e L. Z. MELGAR ${ }^{1}$ \\ ${ }^{1}$ Universidade Federal de São João del-Rei, Departamento de Engenharia Química e \\ Estatística \\ E-mail para contato: fernandaribeirods@hotmail.com
}

\begin{abstract}
RESUMO - O estudo teve como objetivo a realização de um estudo cinético do processo de adsorção do corante aniônico Alaranjado de Metila (AM) em argila bentonita ativada termicamente, a fim de avaliar a natureza desse processo. A argila tratada foi caracterizada por difratometria de raio $\mathrm{X}$ e foi avaliado o efeito da concentração inicial (300-500 mg L $\mathrm{m}^{-1}$ ). O estudo cinético revelou que o modelo de pseudossegunda ordem foi melhor ajustado. A capacidade máxima de adsorção encontrada foi de $313,47 \mathrm{mg} \mathrm{g}^{-1}$. Os resultados revelaram que a argila bentonita é um adsorvente promissor para a retenção do Alaranjado de Metila.
\end{abstract}

\section{INTRODUÇÃO}

Somente a indústria têxtil consome $15 \%$ do volume de água potável mundial, devolvendo-a contaminada ao meio ambiente (QUADROS, 2005). Nesse contexto, as argilas, que são materiais naturais e considerados de baixo custo, estão sendo muito estudadas nos últimos anos como adsorventes alternativos ao carvão ativado na remoção de corantes em efluentes (ROSSETTO et al., 2009), principal resíduo das indústrias têxteis.

Entre as argilas utilizadas como adsorventes destacam-se as esmectitas, que são aquelas constituídas, em sua maioria, por argilominerais esmectíticos, dentre eles a montmorillonita. Essas argilas estão dispostas em estrutura de lamelas ou folhas que são formadas por tetraedros de sílicio e octaedros de alumínio, combinadas em arranjo T-O-T ou 2:1, uma folha octaédrica central com duas folhas tetraédricas nas extremidades que são unidas por ligação covalente. Entre uma lamela e outra existe uma distância denominada distância basal ou interlamelar, onde há a presença de água e cátions metálicos $\left(\mathrm{Na}^{+}, \mathrm{K}^{+}\right.$, $\mathrm{Ca}^{2+}$ ) que podem ser substituídos por outras moléculas (BARBOSA, 2009).

A larga aplicabilidade desse material está associada à elevada área superficial, grande capacidade de troca de cátions (CTC), baixo custo, grande disponibilidade e boa inércia química. Além disso, as argilas bentonitas mostram uma forte afinidade para a adsorção de corantes catiônicos e aniônicos (DUARTE-NETO et al., 2014). 
Para produzir um material com melhor capacidade de retenção de efluentes têxteis é necessária a sua modificação e de suas propriedades físico-químicas. A capacidade dos argilominerais em adsorver água nas regiões interlamelares e expandir, reduz a adsorção de corantes (TEIXEIRA-NETO, TEIXEIRA-NETO, 2009). Para minimizar esse efeito nas argilas realizou-se tratamento térmico a fim de torná-la mais adequada ao processo de adsorção e suas aplicações industriais. Portanto, neste estudo foi utilizada argila bentonita tratada termicamente para a remoção de corante têxtil de soluções aquosas. Foi investigado o efeito da concentração inicial de AM e os resultados foram ajustados aos modelos cinéticos de pseudoprimeira ordem, pseudossegunda ordem e de Weber-Morris.

\section{MATERIAIS E MÉTODOS}

\subsection{Materiais}

Argila utilizada foi do tipo Bentonita sódica natural, cedida pela empresa ALIANÇA LATINA INDÚSTRIA E COMÉRCIO LTDA proveniente da cidade de Uruguaiana/RS. O corante estudado foi Alaranjado de Metila (AM) com grau de pureza de $85 \%(\mathrm{~m} / \mathrm{m})$, fornecido pela empresa DINÂMICA QUÍMICA CONTEMPORÂNEA LTDA.

\subsection{Metodologia Experimental}

Tratamento térmico: A argila in natura foi submetida ao processo de calcinação em Forno Mufla SP Labor, modelo SP-1200, a $500{ }^{\circ} \mathrm{C}$ pelo período de 24 horas e padronizadas em peneira malha 200 mesh.

Caracterização: As amostras foram caracterizadas por difratometria de raio-X em equipamento Miniflex, modelo Rigaku 300/600+, usando radiação de $\operatorname{CuK} \alpha(\lambda=1,5418 \AA)$. Corrente de $15 \mathrm{~mA}$ e tensão de $40 \mathrm{kV}$, com varredura de $5^{\circ}$ a $60^{\circ}(2 \Theta)$ e velocidade de $1^{\circ}$ $\min ^{-1}$.

Teste de adsorção: $200 \mathrm{mg}$ da argila in natura e calcinada foram adicionados a $50 \mathrm{~mL}$ de água destilada e mantidas em contato durante 2 horas. Em seguida, adicionou-se o corante (AM) resultando em solução com concentração de $5,68 \mathrm{mg} / \mathrm{L}, \mathrm{pH} 8$ e mantidos à $25^{\circ} \mathrm{C}$.

Estudo cinético: Os estudos para avaliar a cinética da adsorção foram realizados adicionando argila modificada termicamente $\left(1 \mathrm{~g} \mathrm{~L}^{-1}\right)$ em $50 \mathrm{~mL}$ de solução de corante, mantidos a agitação e temperatura constantes de $200 \mathrm{rpm}$ e $25^{\circ} \mathrm{C}$. O sistema foi monitorado utilizando incubadora Shaker Lucadema, modelo Luca-223, controlando o tempo de contato estabelecido de 180 minutos. Variou-se a concentração inicial (300-500 mg L $\left.{ }^{-1}\right)$ do corante AM. A fim de analisar as concentrações no equilíbrio, as absorbâncias foram medidas utilizando o espectrofotômetro de UV-VIS Micronal, modelo AJX-1600 Spectrophotometer, comprimento de onda de $468 \mathrm{~nm}$.

\subsection{Modelos cinéticos}

O comportamento cinético da adsorção foi obtido por ajuste dos modelos de pseudoprimeira ordem (Equação 1), pseudossegunda ordem (Equação 2) e modelo de WeberMorris (Equação 3) (NASCIMENTO et al., 2014).

$$
\ln \left(q_{e}-q_{t}\right)=\ln \left(q_{e}\right)-k_{1} t
$$




$$
\begin{aligned}
& \frac{t}{q_{t}}=\frac{1}{k_{2} q_{e}^{2}}+\frac{t}{q_{e}} \\
& q_{t e}=K_{d} t^{0,5}+C
\end{aligned}
$$

Onde, $\mathrm{k}_{1}$ : constante da taxa de adsorção de pseudoprimeira ordem $\left(\mathrm{min}^{-1}\right), \mathrm{q}_{\mathrm{e}} \mathrm{e} \mathrm{q}_{\mathrm{t}}$ : quantidades adsorvidas por grama de adsorvente no equilíbrio e no tempo $\mathrm{t}$ (min), respectivamente $\left(\mathrm{mg} \mathrm{g}^{-1}\right), \mathrm{k}_{2}$ : constante da taxa de adsorção de pseudossegunda ordem $\left(\mathrm{g} \mathrm{mg}^{-1}\right.$ $\left.\min ^{-1}\right), \mathrm{q}_{\mathrm{te}}$ : quantidade de adsorvato adsorvida na fase sólida $\left(\mathrm{mg} \mathrm{g}^{-1}\right)$ em um tempo $\mathrm{t}(\mathrm{min})$, $\mathrm{K}_{\mathrm{d}}$ : coeficiente de difusão intrapartícula $\left(\mathrm{mg} \mathrm{g}^{-1} \min ^{-0,5}\right)$, C: constante relacionada com a resistência à difusão $\left(\mathrm{mg} \mathrm{g}^{-1}\right)$.

Os parâmetros cinéticos foram obtidos a partir dos dados de quantidade adsorvida $\mathrm{Q}_{\mathrm{e}}$ $\left(\mathrm{mg} \mathrm{g}^{-1}\right)$, concentração inicial de corante $\mathrm{C}_{0}\left(\mathrm{mg} \mathrm{L}^{-1}\right)$, concentração de equilíbrio na solução $\mathrm{C}_{\mathrm{e}}\left(\mathrm{mg} \mathrm{L}^{-1}\right)$, volume da solução $\mathrm{V}(\mathrm{L})$ e massa de adsorvente $\mathrm{m}$ (g) que podem ser relacionados pela Equação 4 (AUTA e HAMEED, 2014).

$$
Q_{e}=\frac{\left(C_{0}-C_{e}\right) V}{m}
$$

\section{RESULTADOS E DISCUSSÃO}

\subsection{Caracterização}

Na Figura 1, no difratograma de raios $\mathrm{X}$ da argila in natura, pode-se observar que o pico correspondente a $2 \theta=7,04^{\circ}$ relativo à distância interplanar basal do argilomineral Montmorilonita foi da ordem de $12,55 \AA$ diminuiu para $11,20 \AA$ correspondente ao pico $2 \theta=7,88^{\circ}$, após a calcinação. Esse resultado mostrou-se coerente, pois indica a redução do espaçamento basal (d001) após o tratamento térmico, que provocou o colapso da estrutura da argila devido à eliminação de águas de hidratação de cátions interlamelares (NETO et al., 2012). Uma vez que a $500^{\circ} \mathrm{C}$ ocorre a desidratação e desidroxilação do argilomineral provocando a alteração estrutural e perda de massa (ARAUJO et al., 2012).

Figura 1 - Difratograma de raios $\mathrm{X}$ da argila in natura e calcinada.

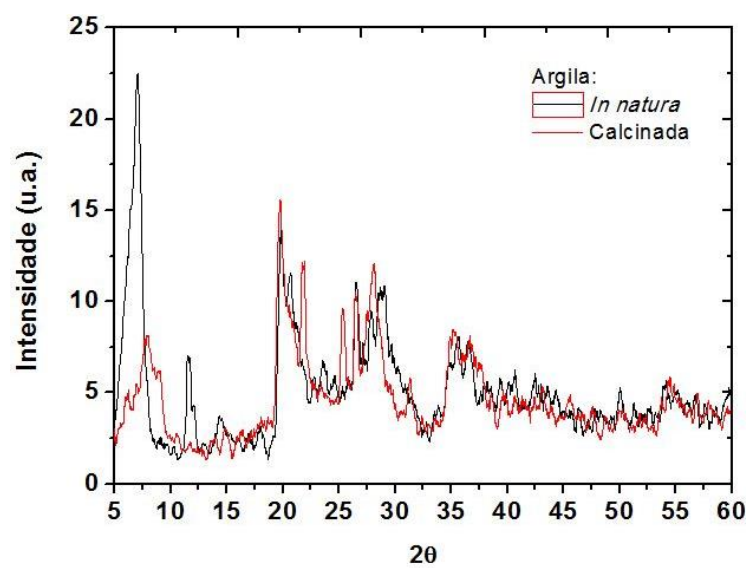




\subsection{Teste de adsorção}

Na Figura 2 pode-se notar que a porcentagem de remoção do AM em argila in natura foi em torno de $10 \%$ enquanto que em argila calcinada foi aproximadamente $40 \%$. Dessa forma, foi evidenciado que o tratamento térmico provocou aumento na capacidade adsortiva do corante aniônico, sugerindo que houve a minimização das cargas negativas interlamelares e, assim, redução da repulsão eletrostática entre corante e adsorvente.

Figura 2 - Estudo da porcentagem de remoção de $\mathrm{AM}\left(5,68 \mathrm{mg} \mathrm{L}^{-1}\right)$ em argila in natura e bentonita calcinada $\left(4 \mathrm{~g} \mathrm{~L}^{-1}\right), \mathrm{pH} 8$, à $25^{\circ} \mathrm{C}$.

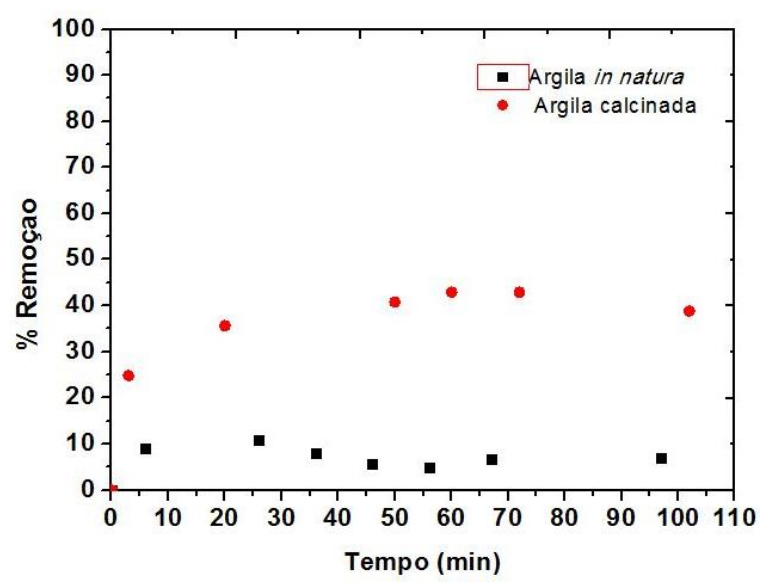

\subsection{Efeito da concentração inicial}

Pela análise do estudo cinético na Figura 3, pode-se perceber que o aumento da concentração inicial favorece a maior adsorção do corante. Isto pode ser explicado pelo fato de que com o aumento da concentração inicial, a força motriz para que a adsorção ocorra aumenta (GOEL et al., 2005), favorecendo a transferência de massa do corante do seio da solução para a superfície do adsorvente. Esse resultado pode ser confirmado pela máxima capacidade de adsorção encontrada de $313,4796 \mathrm{mg} \mathrm{g}^{-1}$ para o ensaio realizado com maior concentração de AM como mostrado na Tabela 1.

Figura 3 - Estudo cinético para avaliar o efeito da variação da concentração inicial na adsorção do $\mathrm{AM}\left(300,400\right.$ e $\left.500 \mathrm{mg} \mathrm{L}^{-1}\right)$ em bentonita calcinada $\left(1 \mathrm{~g} \mathrm{~L}^{-1}\right)$, pH 8, à $25^{\circ} \mathrm{C}$.

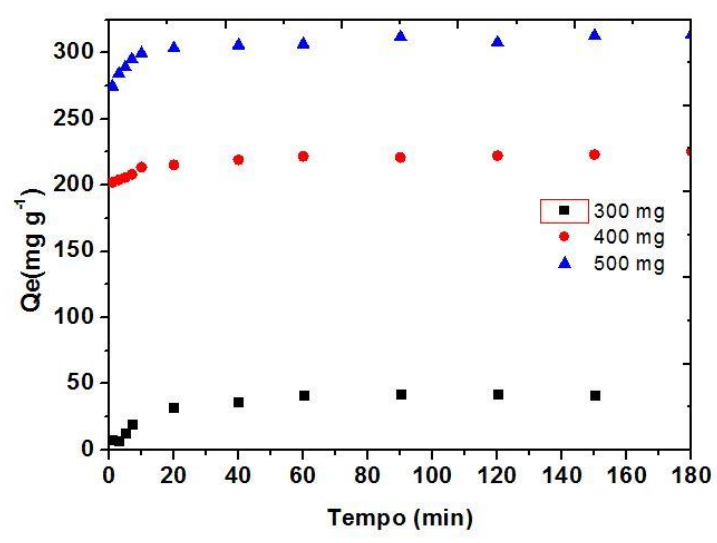


Foi possível constatar também que a adsorção do AM em argila calcinada segue a cinética de pseudossegunda ordem, como demostrado na Tabela 1 , segundo os valores de $\mathrm{R}^{2}$ dessa correlação. Os dados experimentais dos ensaios que utilizaram concentrações de 400 e $500 \mathrm{mg} \mathrm{L}^{-1}$ foram correlacionados pelo modelo de Weber-Morris e, assim, revelam que a etapa limitante é a difusão externa, uma vez que o coeficiente linear da reta (C) encontrado não foi igual a zero (NASCIMENTO, 2014).

Tabela 1 - Valores dos parâmetros dos ajustes aos modelos de pseudoprimeira ordem, pseudossegunda ordem e de Weber-Morris aos dados experimentais obtidos pelos ensaios de adsorção do $\mathrm{AM}$ a $25^{\circ} \mathrm{C}$ e $\mathrm{pH} 8$, em argila calcinada.

\begin{tabular}{|c|c|c|c|}
\hline \multicolumn{4}{|c|}{ Pseudoprimeira ordem } \\
\hline Concentração $\left(\mathrm{mg} \mathrm{L}^{-1}\right)$ & $\mathrm{Q}_{\mathrm{e}}\left(\mathrm{mg} \mathrm{g}^{-1}\right)$ & $\mathrm{K}_{1}\left(\min ^{-1}\right)$ & $\frac{\mathrm{R}^{2}}{2}$ \\
\hline 300 & 297,5582 & 0,00078 & 0,6279 \\
\hline 400 & 206,0893 & 0,00055 & 0,7001 \\
\hline 500 & 258,1988 & 0,00060 & 0,5835 \\
\hline \multicolumn{4}{|c|}{ Pseudossegunda ordem } \\
\hline Concentração $\left(\mathrm{mg} \mathrm{L}^{-1}\right)$ & $\mathrm{Q}_{\mathrm{e}}\left(\mathrm{mg} \mathrm{g}^{-1}\right)$ & $\mathrm{K}_{2}\left(\mathrm{~g} \mathrm{mg}^{-1} \min ^{-1}\right)$ & $\overline{\mathrm{R}^{2}}$ \\
\hline 300 & 45,5580 & 0,00532 & 0,9998 \\
\hline 400 & 224,7191 & 0,00669 & 0,9998 \\
\hline 500 & 313,4796 & 0,00231 & 0,9952 \\
\hline \multicolumn{4}{|c|}{ Weber-Morris } \\
\hline "Concentração $\left(\mathrm{mg} \mathrm{L}^{-1}\right)$ & $\mathrm{CId}\left(\mathrm{mg} \mathrm{g}^{-1} \min ^{-0,5}\right)$ & $\mathrm{C}\left(\mathrm{mg} \mathrm{g}^{-1}\right)$ & $\overline{\mathrm{R}^{2}}$ \\
\hline 300 & 13,4515 & $-16,3646$ & 0,9655 \\
\hline 400 & 2,8769 & 199,2317 & 0,9775 \\
\hline 500 & 11,7435 & 263,0959 & 0,9859 \\
\hline
\end{tabular}

Durante os experimentos foi observado que houve precipitação do corante utilizando concentração $300 \mathrm{mg} \mathrm{L}^{-1}$ em $\mathrm{pH}<5$ e protonação do corante (PREVIDELLO et al., 2006). Dessa forma, os ensaios em $\mathrm{pH}$ mais ácidos, foram impossibilitados, pois não seria possível atribuir a remoção do adsorvato completamente pelo processo de adsorção. A redução da solubilidade do AM em meio ácido ocorre pela reação entre os íons $\mathrm{SO}_{4}^{-2}$ (provenientes da dissociação do ácido sulfúrico em solução aquosa, utilizado para a manutenção do pH baixo) com os cátions livres presentes na região interlamelar da argila. Portanto, ocorre a formação de sais e a presença de eletrólitos fortes reduz a solubilidade do Alaranjado de Metila conforme se aumenta a concentração desse sal (LEAL, 2011). Assim, os ensaios foram todos realizados em $\mathrm{pH}$ básico a fim de evitar a interferência da precipitação no processo adsortivo. $\mathrm{O}$ pH 8 foi escolhido uma vez que obteve-se melhor remoção em comparação ao pH 7.

\section{CONCLUSÃO}

O estudo mostrou que a argila pode ser empregada como adsorvente de corante aniônico em soluções aquosas, sendo eficiente e barato, em comparação a outros métodos de tratamento de efluentes têxteis. Foi possível constatar que o aumento da concentração inicial de corante favoreceu a maior taxa de adsorção. O modelo de pseudossegunda ordem correlacionou melhor ao sistema e encontrou-se a capacidade máxima de adsorção de 313,47 $\mathrm{mg} \mathrm{g}^{-1}$. 


\section{REFERÊNCIAS}

ARAUJO, A.L.P.; NOGUEIRA, I.L.; DA SILVA, M.G.C.; GIMENES, M.L.; DE BARROS, M.A.S.D. Tratamento térmico em argila Bentonita para uso na remoção de zinco em leito fixo. TTT 2012 - VI Conferência Brasileira sobre Temas de Tratamento Térmico Departamento de Engenharia Química Universidade Federal de Maringá e Universidade Estadual de Campinas, Atibaia, SP. 2012.

AUTA, M.; HAMEED, B.H. Chitosan-Clay composite as highly effective and low-cost adsorbent for batch and fixed-bed adsorption for methylene blue. Chemical Engineering Journal - Chemical Engineering Department, College of Engineering King Saud University, Saudi Arabia, n. 237, p. 352-361, 2014.

BARBOSA, E. M. C. Materiais mistos nanoestruturados baseados em argila bentonita e cobalto coloidal. 2009. 56 f. Dissertação (Programa de Pós-Graduação em Química) Universidade Estadual do Centro-Oeste, Paraná. 2009.

DUARTE-NETO, J. F.; CARTAXO, J. M.; NEVES, G. A.; MENEZES, R.R. Processos de adsorção de corantes em argilas esmectíticas: uma revisão. Revista Eletrônica de Materiais e Processos - Departamento de Engenharia de Materiais da Universidade Federal de Campina Grande, Campina Grande, n. 1, p. 51-59, vol. 9. 2014.

GOEL, J.; KADIRVELU, K.; RAJAGOPAL, C.; GARG, V. K. Removal of lead(II) by adsorption using treated granular activated carbon: Batch and column studies. $J$. Hazard. Mater,v. 125, p. 211-220, 2005.

LEAL, C. M. S. Solubilidade de corantes azo. Dissertação de Mestrado em Química Industrial - Universidade da Beira Interior, Covilhã. 2011.

NASCIMENTO, R. F.; LIMA, A. C. A; VIDAL, C. B.; MELO, D. Q.; RAULINO, G. S. C. Adsorção: Aspectos teóricos e aplicações ambientais. Fortaleza: Imprensa Universitária. 2014. 256p.

NETO, A. F. N.; VIEIRA, M. G. A.; DA SILVA, M. G. C. Caracterização térmica de argilas bentoníticas destinadas à remoção de metais. TTT 2012 - VI Conferência Brasileira sobre Temas de Tratamento Térmico. - Faculdade de Engenharia Química Unicamp, Atibaia, SP. 2012.

PREVIDELLO, B. A. F.; CARVALHO F. R.; TESSARO, A. L.; SOUZA, V. R.; HOIKA, N. $\mathrm{O} \mathrm{pK}_{\mathrm{A}}$ de indicadores ácido-base e os efeitos de sistemas coloidais. Quim. Nova Departamento de Química, Universidade Estadual de Maringá, Maringá, Vol. 29, No. 3, 600-606, 2006.

QUADROS, S. S. Tratamento e reutilização de efluentes têxteis gerados nos tingimentos de tecidos de algodão. 2005. 110 f. Dissertação (Programa de Pós-Graduação em Química) - Universidade Regional de Blumenau, Blumenau. 2005.

ROSSETTO, E.; BERALDIN, R.; PENHA, F.G.; PERGHER, S.B.C. Caracterização de argilas bentonitas e diatomitas e sua aplicação como adsorventes. Quim. Nova Departamento de Química, Universidade Regional Integrada do Alto Uruguai e das Missões, Erechim, Vol. 32, No. 8, 2064-2067, 2009.

TEIXEIRA-NETO, E.; TEIXEIRA-NETO, A. Modificação química de argilas: desafios científicos e tecnológicos para obtenção de novos produtos com maior valor agregado. Química Nova - Centro de Ciências Naturais e Humanas da Universidade Federal do ABC, São Paulo, n. 3, p. 809-817, vol. 32. 2009. 